\title{
Major depressive disorder and access to health services among people who use illicit drugs in Vancouver, Canada
}

Tara Beaulieu', Lianping Ti ${ }^{1,4}$, M.-J. Milloy ${ }^{1,2,4}$, Ekaterina Nosova ${ }^{1,2}$, Evan Wood ${ }^{1,2,4}$ and Kanna Hayashi ${ }^{1,2,3^{*}}$ (D)

\begin{abstract}
Background: People who use illicit drugs (PWUD) are commonly diagnosed with major depressive disorder (MDD). However, little is known about whether PWUD living with MDD experience additional barriers to accessing health services compared to those without MDD. We sought to identify whether MDD symptoms were associated with perceived barriers to accessing health services among people who use illicit drugs (PWUD) in Vancouver, Canada.

Methods: Data were collected through prospective cohorts of PWUD in Vancouver, Canada between 2005 and 2016. Using multiple logistic regression, we examined the relationship between MDD symptoms, defined as a Centre for Epidemiologic Studies Depression (CES-D) scale total score of $\geq 16$, and barriers to access health services. We also used descriptive statistics to examine common barriers among participants who reported any barriers.

Results: Among a total of 1529 PWUD, including 521 (34.1\%) females, 415 (27.1\%) reported barriers to accessing health services, and 956 (62.5\%) reported MDD symptoms at baseline. In multiple logistic regression analyses, after adjusting for a range of potential confounders, MDD symptoms (adjusted odds ratio [AOR] $=1.40 ; 95 \%$ confidence interval [CI]: 1.03-1.92) were positively and significantly associated with barriers to accessing health services. Among those who reported MDD symptoms and barriers to access, commonly reported barriers included: long wait lists/ times (38.1\%); and treated poorly by health care professionals (30.0\%).

Conclusion: These findings show that the likelihood of experiencing barriers to accessing health services was higher among PWUD with MDD symptoms compared to their counterparts. Policies and interventions tailored to address these barriers are urgently needed for this subpopulation of PWUD.
\end{abstract}

Keywords: Depression, Access to care, People who use illicit drugs, Canada

\section{Background}

The extent of overlap between major depressive disorder (MDD) and substance use disorder (SUD) is compelling, with an estimated lifetime prevalence of co-occurrence ranging from 27 to $40 \%$ in persons with MDD [1]. There exists notable empirical evidence to shed light on the possible mechanisms underlying SUD-MDD co-occurrence, with the simplest explanation being a bidirectional causal relationship (whether direct or indirect). Other explanations tend to reflect two paths of derivation: genetic,

\footnotetext{
* Correspondence: bccsu-kh@cfenet.ubc.ca

'British Columbia Centre for Excellence in HIV/AIDS, St. Paul's Hospital, 608-1081 Burrard Street, Vancouver, BC V6Z 1Y6, Canada

2British Columbia Centre on Substance Use, St. Paul's Hospital, 1081 Burrard

Street, Vancouver, BC V6Z 1Y6, Canada

Full list of author information is available at the end of the article
}

developmental and environmental factors (e.g., socioeconomic marginalization, early life trauma and a disruptive family environment); or the self-medication hypotheses (i.e., use of illicit drugs to alleviate MDD symptoms) [1-4]. No single or consistent pattern appears to underlie co-occurrence, which lends evidence to suggest that multiple pathways of association may be acting simultaneously.

A growing body of literature has indicated that SUDMDD co-occurrence causes a significant burden to the individual, their families, and society. For instance, this comorbidity is often associated with a greater severity of symptoms, poorer treatment response and enhanced risk for SUD and MDD recurrence [5, 6]. SUD-MDD cooccurrence also yields greater social and personal 
impairments (e.g., interpersonal concerns and reduced occupational functioning), and enhanced risk for comorbid psychiatric conditions, particularly posttraumatic stress disorder (PTSD) and generalized anxiety disorder (GAD) [1]. Moreover, a much higher risk of suicidal ideation and attempt has been evident among this subpopulation [1, 7]. Finally, evidence suggests that there are broader implications in the context of public health and healthcare costs. For example, SUD-MDD co-occurrence often leads to higher rates of acute service utilization (e.g., emergency department and inpatient healthcare utilization) - contributing to higher costs $[8,9]$.

Although there are effective pharmacological and psychotherapeutic options to treat MDD, their efficaciousness is sometimes not well understood among persons with SUD (e.g., when administered concurrently with opioid agonist therapy medications such as methadone or buprenorphine) [10]. Moreover, some prescription drugs have been found to significantly increase harms (e.g., completed suicide) [11]. Additionally, while well-designed psychotherapies and pharmacological treatments for SUD exist, therapeutic efficacy and safety may differ in the presence of MDD co-occurrence [12]. Studies which examine effective treatments for SUD-MDD cooccurrence are sparse and sometimes methodologically, open to doubt. What is clear is that access to care remains disproportionately low among individuals with SUD-MDD co-occurrence [13]. A growing body of literature has explored barriers to SUD and MDD care separately [14-18], and treatment barriers among individuals with SUD and co-occurring mental illness in a broader sense [19]. However, there is a dearth of literature on barriers to care among individuals with SUD-MDD co-occurrence [20]. Therefore, we sought to identify whether MDD symptoms were associated with perceived barriers to accessing health services among people who use illicit drugs (PWUD) in Vancouver, Canada.

\section{Methods}

\section{Study design and population}

Data for this study were drawn from the Vancouver Injection Drug Users Study (VIDUS), and the AIDS Care Cohort to evaluate Exposure to Survival Services (ACCESS), two prospective cohorts involving PWUD in Vancouver, Canada. The methods for these studies have been described elsewhere [21, 22]. To be eligible for VIDUS, participants must be $\geq 18$ years of age, HIVseronegative and report injection drug use at least one month prior to enrollment. ACCESS participants must be $\geq 18$ years of age, HIV-seropositive and report using an illicit drug (other than or in addition to cannabis) in the month prior to enrollment. Recruitment was conducted through self-referral and street outreach.

The data collection instruments and procedures were harmonized across the two cohorts to allow for pooled analyses. At baseline and semi-annually, participants completed an interviewer-administered questionnaire that elicited information on socio-demographic characteristics, drug use patterns, access to healthcare, and other relevant exposures and outcomes. Additionally, at each visit, participants provided blood samples for HIV and HCV serologic tests and HIV disease monitoring as appropriate. A \$30 (CAD) honorarium was offered to participants upon completion of each study visit. The cohorts have received ethical approval by the University of British Columbia/Providence Health Care Research Ethics Board.

\section{Study sample}

For the present analysis, the sample was restricted to those who: 1) completed a baseline between December 2005 and May 2016; and 2) completed at least one follow-up visit directly after baseline to allow for lagged analyses. For those that did not have a Centre for Epidemiologic Studies Depression (CES-D) score at baseline but had one during follow-up, the follow-up was included as the baseline (if at least one subsequent followup visit occurred).

\section{Variable selection}

The primary outcome of interest was having experienced barriers to accessing health services in the last six months, dichotomized as any barriers vs. none. Possible barriers included: 1) Limited hours of operation; 2) Long wait lists/times; 3) Didn't know where to go; 4) Language barrier; 5) Jail/detention/prison; 6) Was treated poorly by health care professionals; 7) Difficulty keeping appointments; 8) Restricted to one physician; 9) No Personal Health Number (PHN); 10) Other (barriers unaccounted for were grouped together for analysis); 11) No barriers, at baseline. The primary explanatory variable was having depressive symptoms, defined as a Centre for Epidemiologic Studies Depression (CES-D) summed score of $\geq 16$. Our analysis utilized this cutpoint as MDD has been typically classified based on a summed score of $\geq 16$ [23-25].

We also considered a selection of possible confounders in the relationship between MDD symptoms and barriers to care, including: age at baseline (per 10year increase); sex (male vs. female); white ethnicity (yes vs. no); calendar year (per one-year increase); HIV serostatus (positive vs. negative); homelessness (yes vs. no); any injection drug use (yes vs. no); daily heroin use (yes vs. no); daily cocaine use (yes vs. no); daily crystal methamphetamine use (yes vs. no); daily prescription opioid 
use (yes vs. no); incarceration (yes vs. no); enrollment in opioid agonist therapy (yes vs. no); hospitalized (yes vs. no); sex work (yes vs. no); stable employment (yes vs. no); history of childhood physical abuse (yes vs. no); history of childhood sexual abuse (yes vs. no); and victim of violence (yes vs. no). With the exception of age, sex, calendar year, HIV serostatus, and history of childhood physical or sexual abuse, all variables were considered as time-updated variables of events reported in the six months prior to interview.

\section{Statistical analyses}

As a first step, we examined descriptive and sociodemographic characteristics of the sample, stratified by having had recently experienced barriers to accessing health services at baseline. Comparisons were made using the Pearson's $\chi^{2}$ test for binary variables and the Mann-Whitney U test for continuous variables. Next, we used bivariate logistic regression to estimate the relationship between the outcome (i.e., barriers to accessing health services in the last six months) and all explanatory variables, including CES-D summed score. Then, a multiple logistic regression model was constructed where all secondary variables significant at $p<0.10$ in bivariate analyses were included. Of note, the explanatory variable and the primary outcome of interest covered the previous two weeks and six months, respectively; thus, the CES-D variable was lagged in response to potential temporal concerns.

In a stepwise manner, we compared the value of the coefficient in the full model to the value of the coefficient for the main relationship in each of the reduced models, the variable associated with the smallest relative change was dropped. We continued this iterative process until the maximum change exceeded $5 \%$. Multicollinearity was assessed using the variance inflation factor. Finally, the following variables were forced back into the final model as these variables are known confounders in the main relationship: sex; homelessness; and injection drug use in the last six months.

As a secondary analysis, we accessed frequencies to determine common barriers to accessing health services among participants who reported 'yes' to the main outcome: in total and stratified by CES-D summed score $(\geq 16$ vs. $<16)$. All analyses were performed in $\mathrm{R}$ version 3.2.4 (Foundation for Statistical Computing, Vienna, Austria).

\section{Results}

Baseline descriptive and socio-demographic characteristics, stratified by the outcome are presented in Table 1. Among a total of 1529 PWUD, 801 (87.3\%) of VIDUS participants and 481 (79.1\%) of ACCESS participants reported injecting drug use in the past six months. 415 (27.1\%) reported barriers to accessing health services and $956(62.5 \%)$ had a CES-D total score $\geq 16$ at baseline. Among those with a CES-D total score $\geq 16$ at baseline, 297 (71.6\%) reported barriers to accessing health services whereas 650 (59.1\%) reported no barriers to accessing health services, resulting in a difference of $12.5 \%$. Five hundred twenty-one $(34.1 \%)$ were female, $878(57.4 \%)$ had white ancestry, and the median age at baseline was 43 years (quartile [Q]1 - Q3: 36-49 years). The median difference between the lagged date for the explanatory variable and current date for the outcome variable was 6 months (Q1 - Q3: 6-6).

As presented in Table 2, in a multiple logistic regression analyses after adjusting for a range of confounders, lagged CES-D total score $\geq 16$ remained independently associated with barriers to accessing health services (adjusted odds ratio $[\mathrm{AOR}]=1.40 ; 95 \%$ confidence interval [CI]: 1.03-1.92). The relative risk (RR) was 1.20 (95\% CI, 1.12-1.29). Suffered physical violence also remained independently associated with barriers to accessing health services $(\mathrm{AOR}=1.38$; 95\% CI: 1.01-1.86). Among those who reported a CES-D total score of $\geq 16$ and barriers to accessing health services, the most commonly reported barriers included: long wait lists/times (38.1\%); treated poorly by health care professionals (30.0\%); and difficulty keeping appointments (18.5\%). Among those who reported a CES-D total score $<16$ and barriers to accessing health services, the most commonly reported barriers included: long wait lists/times (30.3\%); treated poorly by health care professionals (27.0\%); and difficulty keeping appointments (14.6\%).

\section{Discussion}

In the present study, more than half of PWUD showed MDD symptoms. We observed a high proportion of participants who reported barriers to accessing health services. We also found a positive and independent relationship between MDD and barriers to accessing health services among PWUD, after adjusting for various confounders.

While these results corroborate the findings from previous research in that we found a high prevalence of barriers to care among PWUD [19, 20, 26], there are some inconsistencies between our results and previous findings, which are likely attributable to differences in study environments. For example, in a study conducted in the United States (US), the sample was comprised of 393 patients who met criteria for both MDD and SUD (i.e., 100\% prevalence of MDD-SUD co-occurrence) [20]. Our study sample was comprised of 1529 PWUD, 573 (37.5\%) of which reported no MDD symptoms. Furthermore, this US-based study focused primarily on the role of 
Table 1 Baseline characteristics stratified by having had recently experienced barriers to accessing health services among people who use illicit drugs in Vancouver, Canada $(N=1529)$

\begin{tabular}{lll} 
Total \\
$n=1529, N(\%)$ & Barriers to accessing health services in the last six months & $p-$ value \\
\cline { 2 - 3 } & $\begin{array}{l}\text { Yes } \\
n=415, N(\%)\end{array}$ & \\
& $n=1099, N(\%)$
\end{tabular}

Characteristic

\section{CES-D total score}

$\geq 16$

$<16$

$\mathrm{Age}^{\mathrm{a}}$

median

IQR

Gender

male

female

Caucasian ethnicity

yes

no

Calendar year

median

IQR

HIV serostatus

positive

negative

Homelessness $^{b}$

yes

no

Any injection drug use ${ }^{b}$

$$
\text { yes }
$$

no

Daily heroin use $\mathrm{b}^{\mathrm{b}}$

$$
\text { yes }
$$

no

Daily cocaine use $\mathrm{b}^{\mathrm{b}}$

$$
\text { yes }
$$

no

Daily crystal methamphetamine use $\mathrm{b}^{\mathrm{b}}$

$$
\text { yes }
$$

no

Daily prescription opioid use $\mathrm{b}^{\mathrm{b}}$

$$
\text { yes }
$$

no

Incarceration $^{\mathrm{b}}$

$$
\text { yes }
$$

no
$956(62.5)$

459 (30)

43.0

(36.2-48.6)

1008 (65.9)

$521(34.1)$

$878(57.4)$

$651(42.6)$

2007

(2006-2009)

2007

(2006-2009)

147 (35.4)

$268(64.6)$

$164(39.5)$

$246(59.3)$

1041 (68.1)

1282 (83.8)

378 (91.1)

$37(8.9)$

107 (25.8)

$308(74.2)$

$346(22.6)$

$1179(77.1)$

$130(8.5)$

49 (11.8)

1393 (91.1)

$365(88.0)$

74 (4.8)

1450 (94.8)

$28(6.7)$

387 (93.3)

97 (6.3)

1429 (93.5)

$39(9.4)$

376 (90.6)

228 (14.9)

72 (17.3)

340 (81.9)
650 (59.1)

364 (33.1)

43.1

0.119

(36.6-49.0)

739 (67.2)

0.063

$360(32.8)$

624 (56.8)

0.295

475 (43.2)

2007

0.776

(2006-2009)

445 (40.5)

0.071

654 (59.5)

311 (28.3)

$<0.001$

786 (71.5)

891 (81.1)

$<0.001$

206 (18.7)

234 (21.3)

0.066

$862(78.4)$

81 (7.4)

0.006

1014 (92.3)

$44(4.0)$

0.026

1051 (95.6)

$58(5.3)$

0.004

1039 (94.5)

153 (13.9)

0.084 
Table 1 Baseline characteristics stratified by having had recently experienced barriers to accessing health services among people who use illicit drugs in Vancouver, Canada ( $N=1529)$ (Continued)

\begin{tabular}{|c|c|c|c|c|}
\hline & \multirow{2}{*}{$\begin{array}{l}\text { Total } \\
n=1529, \mathrm{~N}(\%)\end{array}$} & \multicolumn{2}{|c|}{ Barriers to accessing health services in the last six months } & \multirow[t]{2}{*}{$p-$ value } \\
\hline & & $\begin{array}{l}\text { Yes } \\
n=415, N(\%)\end{array}$ & $\begin{array}{l}\text { No } \\
n=1099, N(\%)\end{array}$ & \\
\hline \multicolumn{5}{|c|}{ Enrollment in opioid agonist therapy ${ }^{b}$} \\
\hline yes & $667(43.6)$ & $181(43.6)$ & $481(43.8)$ & \multirow[t]{2}{*}{0.994} \\
\hline no & $858(56.1)$ & $232(55.9)$ & $616(56.1)$ & \\
\hline \multicolumn{5}{|c|}{ Hospitalized $^{\mathrm{b}}$} \\
\hline yes & $302(19.8)$ & $91(21.9)$ & $208(18.9)$ & \multirow[t]{2}{*}{0.191} \\
\hline no & $1227(80.2)$ & $324(78.1)$ & $891(81.1)$ & \\
\hline \multicolumn{5}{|c|}{ Sex work ${ }^{\mathrm{b}}$} \\
\hline yes & $222(14.5)$ & $71(17.1)$ & $148(13.5)$ & \multirow[t]{2}{*}{0.064} \\
\hline no & $1300(85.0)$ & $340(81.9)$ & $948(86.3)$ & \\
\hline \multicolumn{5}{|c|}{ Stable employment ${ }^{\mathrm{b}}$} \\
\hline yes & $371(24.3)$ & $94(22.7)$ & $275(25.0)$ & \multirow[t]{2}{*}{0.338} \\
\hline no & $1158(75.7)$ & $321(77.3)$ & $824(75.0)$ & \\
\hline \multicolumn{5}{|c|}{ History of childhood physical abuse } \\
\hline yes & $1061(69.4)$ & $296(71.3)$ & $757(68.9)$ & \multirow[t]{2}{*}{0.076} \\
\hline no & $410(26.8)$ & $95(22.9)$ & $309(28.1)$ & \\
\hline \multicolumn{5}{|c|}{ History of childhood sexual abuse } \\
\hline yes & $655(42.8)$ & $207(49.9)$ & $444(40.4)$ & \multirow[t]{2}{*}{$<0.001$} \\
\hline no & $824(53.9)$ & $192(46.3)$ & $621(56.5)$ & \\
\hline \multicolumn{5}{|c|}{ Victim of violence $^{b}$} \\
\hline yes & $324(21.2)$ & $125(30.1)$ & $195(17.7)$ & \multirow[t]{2}{*}{$<0.001$} \\
\hline no & $1200(78.5)$ & $288(69.4)$ & $901(82.0)$ & \\
\hline
\end{tabular}

aper 10-year increase

${ }^{\mathrm{b}}$ Activities reported in the six months prior to interview

financial barriers to accessing SUD and evidencebased MDD care. Our study provides new insight into client perspectives in a setting where there are less financial barriers to SUD or evidence-based MDD care due to universal health care. That being said, it is important to consider that British Columbia's provincial health insurance program covers limited mental health care.

In line with previous findings, we found a relationship between MDD and barriers to care among PWUD [20]. Encouragingly, MDD symptoms in PWUD often improve following SUD treatment initiation [5, 27]. Given the central role of primary care physicians in SUD care in Canada, and abroad [13, 28], it is likely that the integration of SUD care into primary care settings would lead to improved depressive symptoms for many. In circumstances where MDD is not a self-limited condition, integration of evidence-based MDD care with primary care could also potentially reduce wait times, improve continuity of care, and increase patient satisfaction [29, 30]. Research data suggest scaling-up collaborative and integrated care pathways as another potential approach to reduce wait times while improving continuity and satisfaction with care among individuals with SUD-MDD co-occurrence [31, 32].

The perception of being 'treated poorly by health care professionals' may be a consequence of mental illnessrelated stigma, substance use-related stigma, and/or abstinence-only based policies within healthcare settings. Our findings underscore a widely prevalent perception of poor treatment by health care professionals among PWUD, regardless of the presence of MDD symptoms [33]. Empirical evidence suggests that a certain level of "mutual mistrust" can exist between providers and PWUD. This may be partially attributable to the fact that providers who are not specialized in addiction medicine lack confidence in providing care for those living with SUD. This can lead to inconsistency in care or avoidance by the provider, which could be interpreted as a sign of maltreatment by PWUD [34]. Provider training such as addiction medicine programs which have sought to integrate substance use care with the rest of medicine, anti-stigma interventions, contact-based education approaches which enhance provider comfort and skills 
Table 2 Multiple logistic regression model to determine the relationship between CES-D score and barriers to accessing health services among people who use illicit drugs in Vancouver, Canada $(n=1529)$

\begin{tabular}{|c|c|c|c|c|}
\hline \multirow[b]{2}{*}{ Characteristic } & \multicolumn{2}{|l|}{ Unadjusted } & \multicolumn{2}{|l|}{ Adjusted } \\
\hline & Odds ratio $(95 \% \mathrm{Cl})$ & $p$-value & Odds ratio $(95 \% \mathrm{Cl})$ & $p$-value \\
\hline \multicolumn{5}{|l|}{ CES-D total score ${ }^{a}$} \\
\hline$(\geq 16$ vs. $<16)$ & $1.63(1.23-2.18)$ & 0.001 & $1.40(1.03-1.92)$ & 0.035 \\
\hline \multicolumn{5}{|l|}{ Age } \\
\hline (per 10-year increase) & $0.81(0.70-0.93)$ & 0.002 & $0.88(0.76-1.02)$ & 0.096 \\
\hline \multicolumn{5}{|l|}{ Gender } \\
\hline (male vs. female) & $0.85(0.66-1.10)$ & 0.214 & $0.99(0.74-1.31)$ & 0.927 \\
\hline \multicolumn{5}{|l|}{ Caucasian ethnicity } \\
\hline (yes vs. no) & $1.02(0.80-1.31)$ & 0.869 & & \\
\hline \multicolumn{5}{|l|}{ Calendar year } \\
\hline (per 1-year increase) & $0.99(0.94-1.03)$ & 0.564 & & \\
\hline \multicolumn{5}{|l|}{ HIV serostatus } \\
\hline (positive vs. negative) & $0.82(0.64-1.06)$ & 0.139 & & \\
\hline \multicolumn{5}{|l|}{ Homelessness $^{\mathrm{b}}$} \\
\hline (yes vs. no) & $1.31(1.00-1.72)$ & 0.049 & $1.11(0.83-1.49)$ & 0.467 \\
\hline \multicolumn{5}{|l|}{ Any injection drug use ${ }^{\mathrm{b}}$} \\
\hline (yes vs. no) & $1.37(1.03-1.84)$ & 0.032 & $1.21(0.89-1.65)$ & 0.224 \\
\hline \multicolumn{5}{|l|}{ Daily heroin use $\mathrm{e}^{\mathrm{b}}$} \\
\hline (yes vs. no) & $1.13(0.84-1.52)$ & 0.419 & & \\
\hline \multicolumn{5}{|l|}{ Daily cocaine use $\mathrm{b}^{\mathrm{b}}$} \\
\hline (yes vs. no) & $0.87(0.53-1.35)$ & 0.543 & & \\
\hline \multicolumn{5}{|c|}{ Daily crystal methamphetamine use $\mathrm{e}^{\mathrm{b}}$} \\
\hline (yes vs. no) & $1.80(1.05-3.01)$ & 0.027 & & \\
\hline \multicolumn{5}{|c|}{ Daily prescription opioid use $\mathrm{e}^{\mathrm{b}}$} \\
\hline (yes vs. no) & $1.44(0.87-2.32)$ & 0.140 & & \\
\hline \multicolumn{5}{|l|}{ Incarceration $^{b}$} \\
\hline (yes vs. no) & $1.72(1.22-2.41)$ & 0.002 & & \\
\hline \multicolumn{5}{|c|}{ Enrollment in opioid agonist therapy ${ }^{\mathrm{b}}$} \\
\hline (yes vs. no) & $0.92(0.72-1.18)$ & 0.511 & & \\
\hline \multicolumn{5}{|l|}{ Hospitalized $^{\mathrm{b}}$} \\
\hline (yes vs. no) & $1.67(1.22-2.27)$ & 0.001 & & \\
\hline \multicolumn{5}{|l|}{ Sex work ${ }^{b}$} \\
\hline (yes vs. no) & $1.08(0.74-1.54)$ & 0.689 & & \\
\hline \multicolumn{5}{|l|}{ Stable employment ${ }^{\mathrm{b}}$} \\
\hline (yes vs. no) & $0.81(0.59-1.10)$ & 0.190 & & \\
\hline \multicolumn{5}{|c|}{ History of childhood physical abuse } \\
\hline (yes vs. no) & $1.47(1.09-1.99)$ & 0.012 & & \\
\hline \multicolumn{5}{|c|}{ History of childhood sexual abuse } \\
\hline (yes vs. no) & $1.44(1.12-1.86)$ & 0.004 & $1.30(0.99-1.70)$ & 0.060 \\
\hline \multicolumn{5}{|l|}{ Victim of violence ${ }^{b}$} \\
\hline (yes vs. no) & $1.55(1.16-2.07)$ & 0.003 & $1.38(1.01-1.86)$ & 0.039 \\
\hline
\end{tabular}

CES-D Center for Epidemiological Studies Depression Scale, CI Confidence Interval

${ }^{\mathrm{b}}$ Activities reported in the six months prior to interview 
in treating those with MDD and/or SUD, and efforts to abolish abstinence-only based policies in healthcare settings may help to address this barrier [30, 35]. Future research should explore the impact of these diverse and innovative strategies to improve MDD and SUD diagnosis, uptake and continuity of care among PWUD.

Our findings should be viewed in the context of several limitations. First, measurement of the primary explanatory variable and the outcome were reliant on self-reported data, which is susceptible to social desirability and recall bias. Second, as with all MDD screening measures, there are psychometric limitations of the CES-D [36, 37]. However, the CES-D has demonstrated validity and reliability in numerous populations (including among PWUD), and has been used extensively in epidemiologic research [38-40]. Third, we relied on a lagged explanatory variable; therefore, there may have been a time gap between when the explanatory and outcome variables were measured. However, we found that the median time between these variables was 6 months. Given that the median duration of an MDD episode is approximately 20 weeks, we decided to use a lagged explanatory variable in our analyses. Forth, despite adjusting models for known confounders, results may be subject to underlying residual and unmeasured confounding. Fifth, these data are observational, thus we were unable to draw conclusions regarding causality. Finally, participants were not randomly selected and therefore, the extent to which these results may be generalized beyond our setting is unclear.

\section{Conclusion}

Overall, our findings demonstrate an essential need for scaling-up access to evidence-based SUD and MDD care among PWUD populations. The integration of evidencebased SUD and MDD care with primary care, scaling-up collaborative and integrated care pathways (to diminish long wait lists/times), provider education, anti-stigma interventions, and efforts to extinguish abstinence-only based policies in healthcare settings (to diminish perceived stigma by health care professionals) may help to alleviate MDD and/or SUD symptoms among PWUD populations. Although future studies are required to fully elucidate the impact of SUD-MDD co-occurrence and to provide guidance for healthcare providers facing the challenge of treating SUD-MDD co-occurrence, the results of this analysis clearly indicate that policies and interventions tailored to address these barriers are urgently needed to mitigate the alarming rates of morbidity and mortality experienced among individuals with SUD-MDD co-occurrence.

\section{Abbreviations}

ACCESS: AIDS Care Cohort to evaluate Exposure to Survival Services; AOR: Adjusted odds ratio; CES-D: Centre for Epidemiologic Studies Depression; Cl: Confidence interval; GAD: Generalized anxiety disorder; MDD: Major depressive disorder; PHN: Personal Health Number; PTSD: Posttraumatic stress disorder; PWUD: People who use illicit drugs; Q: Quartile; US: United States; VIDUS: Vancouver Injection Drug Users Study

\section{Acknowledgements}

The authors thank the study participants for their contribution to the research, as well as current and past researchers and staff.

\section{Funding}

This work was supported by the US National Institutes of Health (VIDUS: U01DA038886; ACCESS: U01DA021525) This research was undertaken, in part, thanks to funding from the Canada Research Chairs program through a Tier 1 Canada Research Chair in Inner City Medicine which supports Dr. Evan Wood. Dr. Lianping Ti is supported by a Michael Smith Foundation for Health Research (MSFHR) Scholar Award. Dr. Kanna Hayashi is supported by a Canadian Institutes of Health Research (CIHR) New Investigator Award (MSH-141971) and MSFHR Scholar Award. Dr. M-J Milloy is supported by a CIHR New Investigator Award, an MSFHR Scholar Award and the US NIH (U01DA0251525). His institution has received an unstructured gift from NG Biomed, Ltd., to support his research.

\section{Availability of data and materials}

The cohort data is not publicly available. For further information regarding the data and materials used please contact the corresponding author.

\section{Authors' contributions}

$L T, K H$, and $T B$ designed the study and the present analysis plan. EN conducted the statistical analyses. TB drafted the manuscript, and incorporated suggestions from all co-authors. All authors made significant contributions to drafting of the manuscript. All authors read and approved the final manuscript.

Ethics approval and consent to participate

Participants from VIDUS and ACCESS cohorts have provided informed consent. The cohorts have received ethical approval by the University of British Columbia/Providence Health Care Research Ethics Board.

Consent for publication

Not applicable

Competing interests

The authors declare that they have no competing interests.

\section{Publisher's Note}

Springer Nature remains neutral with regard to jurisdictional claims in published maps and institutional affiliations.

\section{Author details}

${ }^{1}$ British Columbia Centre for Excellence in HIV/AIDS, St. Paul's Hospital, 608-1081 Burrard Street, Vancouver, BC V6Z 1Y6, Canada. 'British Columbia Centre on Substance Use, St. Paul's Hospital, 1081 Burrard Street, Vancouver, BC V6Z 1Y6, Canada. ${ }^{3}$ Faculty of Health Sciences, Simon Fraser University, Blusson Hall, 8888 University Drive, Burnaby, BC V5A 156, Canada. ${ }^{4}$ Department of Medicine, University of British Columbia, 2775 Laurel Street, Vancouver, BC V5Z 1M9, Canada.

Received: 14 November 2017 Accepted: 12 January 2018 Published online: 19 January 2018

\section{References}

1. Davis L, Uezato A, Newell JM, Frazier E. Major depression and comorbid substance use disorders. Curr Opin Psychiatry. 2008;21(1):14-8.

2. Swendsen JD, Merikangas KR. The comorbidity of depression and substance use disorders. Clin Psychol Rev. 2000;20(2):173-89.

3. Lo CC, Cheng TC, de la Rosa IA. Depression and substance use: a temporal-ordered model. Subst Use Misuse. 2015;50(10):1274-83. 
4. Ng E, Browne CJ, Samsom JN, AHC W. Depression and substance use comorbidity: what we have learned from animal studies. Am J Drug Alcohol Abuse. 2017;43(4):456-74.

5. Rounsaville BJ, Weissman MM, Crits-Christoph K, Wilber C, Kleber H. Diagnosis and symptoms of depression in opiate addicts. Course and relationship to treatment outcome. Arch Gen Psychiatry. 1982;39(2):151-6.

6. Hunter SB, Watkins KE, Hepner KA, et al. Treating depression and substance use: a randomized controlled trial. J Subst Abus Treat. 2012;43(2):137-51.

7. Currie SR, Patten SB, Williams JVA, et al. Comorbidity of major depression with substance use disorders. Can J Psychiatr Rev Can Psychiatr. 2005;50(10):660-6.

8. RachBeisel J, Scott J, Dixon L. Co-occurring severe mental illness and substance use disorders: a review of recent research. Psychiatr Serv. 1999; 50(11):1427-34.

9. Valderas JM, Starfield B, Sibbald B, Salisbury C, Roland M. Defining Comorbidity: implications for understanding health and health services. Ann Fam Med. 2009;7(4):357-63.

10. Hassan AN, Howe AS, Samokhvalov AV, Le Foll B, George TP. Management of mood and anxiety disorders in patients receiving opioid agonist therapy: review and meta-analysis. Am J Addict. 2017;26(6):551-63.

11. Le Noury J, Nardo JM, Healy D, et al. Restoring study 329: efficacy and harms of paroxetine and imipramine in treatment of major depression in adolescence. BMJ. 2015;351:h4320.

12. Beaulieu S, Saury S, Sareen J, et al. The Canadian Network for Mood and Anxiety Treatments (CANMAT) task force recommendations for the management of patients with mood disorders and comorbid substance use disorders. Ann Clin Psychiatry. 2012;24(1):38-55.

13. Urbanoski KA, Rush BR, Wild TC, Bassani DG, Castel S. Use of mental health care services by Canadians with co-occurring substance dependence and mental disorders. Psychiatr Serv. 2007;58(7):962-9.

14. Mojtabai R, Olfson M, Sampson NA, et al. Barriers to mental health treatment: results from the National Comorbidity Survey Replication. Psychol Med. 2011;41(8):1751-61.

15. Reddy MS. Depression: the disorder and the burden. Indian J Psychol Med. 2010;32(1):1-2

16. Sareen J, Jagdeo A, Cox BJ, et al. Perceived barriers to mental health service utilization in the United States, Ontario, and the Netherlands. Psychiatr Serv. 2007:58(3):357-64.

17. Andrade LH, Alonso J, Mneimneh Z, et al. Barriers to mental health treatment: results from the WHO World Mental Health surveys [Internet] Psychol Med. 2014. [cited 2017 Sept 18]; Available from: /core/journals/ psychological-medicine/article/barriers-to-mental-health-treatment-resultsfrom-the-who-world-mental-health-surveys/ 8779313B29B9F3950A0A1154949E0D21.

18. Browne T, Priester MA, Clone S, lachini A, DeHart D, Hock R. Barriers and facilitators to substance use treatment in the rural south: a qualitative study. J Rural Health. 2016;32(1):92-101.

19. Priester MA, Browne T, lachini A, Clone S, DeHart D, Seay KD. Treatment access barriers and disparities among individuals with co-occurring mental health and substance use disorders: an integrative literature review. J Subst Abus Treat. 2016;61:47.

20. Mojtabai R, Chen L-Y, Kaufmann CN, Crum RM. Comparing barriers to mental health treatment and substance use disorder treatment among individuals with comorbid major depression and substance use disorders. J Subst Abus Treat. 2014;46(2):268-73.

21. Strathdee SA, Palepu A, Cornelisse PGA, et al. Barriers to use of free antiretroviral therapy in injection drug users. JAMA. 1998;280(6):547-9.

22. Wood E, Tyndall MW, Spittal PM, et al. Unsafe injection practices in a cohort of injection drug users in Vancouver: could safer injecting rooms help? CMAJ. 2001;165(4):405-10.

23. The CES-D ScaleApplied Psychological Measurement - Lenore Sawyer Radloff. 1977 [Internet]. [cited 2017 Sept 18]; Available from: http://journals. sagepub.com/doi/pdf/10.1177/014662167700100306.

24. Janssen I, Powell LH, Matthews KA, et al. Relation of persistent depressive symptoms to coronary artery calcification in women aged 46 to 59 years. Am J Cardiol. 2016;117(12):1884-9.

25. Wada K, Tanaka K, Theriault G, et al. Validity of the Center for Epidemiologic Studies Depression Scale as a screening instrument of major depressive disorder among Japanese workers. Am J Ind Med. 2007;50(1):8-12.

26. Urbanoski KA, Cairney J, Bassani DG, Rush BR. Perceived unmet need for mental health care for Canadians with co-occurring mental and substance use disorders. Psychiatr Serv. 2008;59(3):283-9.
27. Gossop M, Marsden J, Stewart D. Remission of psychiatric symptoms among drug misusers after drug dependence treatment. J Nerv Ment Dis. 2006; 194(11):826-32.

28. Padwa H, Urada D, Antonini VP, Ober A, Crèvecoeur-MacPhail DA, Rawson RA. Integrating substance use disorder services with primary care: the experience in California. J Psychoactive Drugs. 2012:44(4):299-306.

29. Rothman AA, Wagner EH. Chronic illness management: what is the role of primary care? Ann Intern Med. 2003;138(3):256-61.

30. Beaulieu T, Patten S, Knaak S, Weinerman R, Campbell H, Lauria-Horner B. Impact of skill-based approaches in reducing stigma in primary care physicians: results from a double-blind, parallel-cluster, randomized controlled trial. Can J Psychiatr. 2017;62(5):327-35.

31. Best Practices: Developing Standards of Care for Individuals With Cooccurring Psychiatric and Substance Use Disorders | Psychiatric Services [Internet]. [cited 2017 Sept 18]; Available from: http://ps.psychiatryonline. org/doi/abs/10.1176/appi.ps.52.5.597.

32. The Evolution of Collaborative Mental Health Care in Canada: A Shared Vision for the Future - ProQuest [Internet]. [cited 2017 Sept 22]; Available from: https://search.proquest.com/openview/ ac21342c15af2c8eff2b633859a870f1/1?pq-origsite=gscholar\&cbl=35547.

33. van Boekel LC, Brouwers EPM, van Weeghel J, Garretsen HFL. Stigma among health professionals towards patients with substance use disorders and its consequences for healthcare delivery: systematic review. Drug Alcohol Depend. 2013;131(1-2):23-35.

34. Merrill JO, Rhodes LA, Deyo RA, Marlatt GA, Bradley KA. Mutual mistrust in the medical Care of Drug Users. J Gen Intern Med. 2002;17(5):327-33.

35. Rasyidi E, Wilkins JN, Danovitch I. Training the next generation of providers in addiction medicine. Psychiatr Clin North Am. 2012;35(2):461-80.

36. Gay CL, Kottorp A, Lerdal A, Lee KA. Psychometric Limitations of the Center for Epidemiologic Studies-Depression Scale for Assessing Depressive Symptoms among Adults with HIV/AIDS: A Rasch Analysis [Internet]. Depress Res Treat. 2016. [cited 2017 Sept 9]; Available from: https://www. hindawi.com/journals/drt/2016/2824595/.

37. Morin AJS, Moullec G, Maïano C, Layet L, Just J-L, Ninot G. Psychometric properties of the Center for Epidemiologic Studies Depression Scale (CES-D) in French clinical and nonclinical adults. Rev Epidemiol Sante Publique. 2011:59(5):327-40

38. Golub ET, Latka M, Hagan $\mathrm{H}$, et al. Screening for depressive symptoms among HCV-infected injection drug users: examination of the utility of the CES-D and the Beck depression inventory. J urban health. Bull N Y Acad Med. 2004;81(2):278-90.

39. Zhang W, O'Brien N, Forrest Jl, et al. Validating a shortened depression scale (10 item CES-D) among HIV-positive people in British Columbia, Canada. PLoS One. 2012;7(7):e40793.

40. Hann D, Winter K, Jacobsen P. Measurement of depressive symptoms in cancer patients: evaluation of the Center for Epidemiological Studies Depression Scale (CES-D). J Psychosom Res. 1999;46(5):437-43.

\section{Submit your next manuscript to BioMed Central and we will help you at every step:}

- We accept pre-submission inquiries

- Our selector tool helps you to find the most relevant journal

- We provide round the clock customer support

- Convenient online submission

- Thorough peer review

- Inclusion in PubMed and all major indexing services

- Maximum visibility for your research

Submit your manuscript at www.biomedcentral.com/submit 